\title{
ON THE QUESTION OF GLOBAL EXISTENCE FOR REACTION-DIFFUSION SYSTEMS WITH MIXED BOUNDARY CONDITIONS
}

\author{
BY \\ SELWYN L. HOLLIS ${ }^{1}$ \\ Embry-Riddle Aeronautical University, Daytona Beach, Florida
}

\begin{abstract}
The question of global existence for solutions of reaction-diffusion systems presents fundamental difficulties in the case in which some components of the system satisfy Neumann boundary conditions while others satisfy nonhomogeneous Dirichlet boundary conditions. We discuss particular examples for which classical solutions are known to exist globally when all components satisfy the same type of boundary condition and yet either finite-time blowup occurs or else global existence is unknown when mixed boundary condition types are imposed on the system. Some positive results are presented concerning global existence in the presence of mixed boundary conditions if certain structure requirements are placed on the system, and these results are applied to some particular chemical reaction models.
\end{abstract}

1. Introduction. Recent years have seen a great deal of research and considerable progress on the problem of global existence for systems of weakly coupled, semilinear, parabolic partial differential equations, namely, systems of the form

$$
\begin{array}{ll}
U_{t}-D \Delta U=F(U) & \text { in } \Omega \times\{t>0\}, \\
B U=\beta & \text { on } \partial \Omega \times\{t>0\}, \\
U(\cdot, 0)=U_{0}(\cdot) & \text { on } \Omega
\end{array}
$$

where $U=\left(U_{j}\right)_{j=1}^{m}, F=\left(F_{j}\right)_{j=1}^{m}, D=\operatorname{diag}\left\{d_{1}, d_{2}, \ldots, d_{m}\right\}$ with each $d_{j}>0, \Delta$ is the Laplacian, $\beta=\left(\beta_{j}\right)_{j=1}^{m}, B U=\left(\lambda_{j} U_{j}+\left(1-\lambda_{j}\right) \partial U_{j} / \partial n\right)_{j=1}^{m}$ with $\lambda_{j} \in[0,1]$, $U_{0}=\left(U_{0 j}\right)_{j=1}^{m}$, and $\Omega$ is a bounded domain in $\mathbf{R}^{n}$ with smooth boundary $\partial \Omega$. The following are standard, basic assumptions:

(i) $F: \mathbf{R}_{+}^{m} \rightarrow \mathbf{R}^{m}$ is locally Lipschitz;

(ii) $F_{j}(\zeta) \geq 0$ for all $\zeta \in \mathbf{R}_{+}^{m}$ with $\zeta_{j}=0$;

(iii) $\beta \in C\left(\partial \Omega ; \mathbf{R}_{+}^{m}\right)$;

(iv) $U_{0} \in \boldsymbol{L}^{\infty}\left(\Omega ; \mathbf{R}_{+}^{m}\right)$.

Received April 17, 1991.

1991 Mathematics Subject Classification. Primary 35K45, 35B35.

${ }^{1}$ Current address: Department of Mathematics and Computer Science, Armstrong State College, Savannah, Georgia 31419 USA.

(C)1993 Brown University 
Under these assumptions, the following local existence result is well known.

Proposition 1.1. The system (1.1) admits a nonnegative, noncontinuable, classical solution $U: \Omega \times\left[0, T_{\max }\right) \rightarrow \mathbf{R}_{+}^{m}$ where $0<T_{\max } \leq \infty$. If $T_{\max }<\infty$ then $\|U(\cdot, t)\|_{\infty, \Omega} \rightarrow \infty$ as $t \rightarrow T_{\max }$.

In many cases, it is straightforward to establish that $T_{\max }=\infty$ by means of invariant regions, differential inequalities, etc., but many systems (arising, for example, in modelling chemical reactions) do not lend themselves to these standard methods, and thus a priori bounds must be established by other means in order to deduce that $T_{\max }=\infty$. Two-component systems of the type

$$
\begin{gathered}
u_{t}-d_{1} \Delta u=-u f(v), \quad v_{t}-d_{2} \Delta v=u f(v) \quad \text { in } \Omega \times\{t>0\}, \\
\lambda_{1} u+\left(1-\lambda_{1}\right) \frac{\partial u}{\partial n}=\beta_{1}, \quad \lambda_{2} v+\left(1-\lambda_{2}\right) \frac{\partial v}{\partial n}=\beta_{2} \quad \text { on } \partial \Omega \times\{t>0\}, \\
u(\cdot, 0)=u_{0}, \quad v(\cdot, 0)=v_{0} \quad \text { on } \Omega,
\end{gathered}
$$

where $f \in C^{1}\left(\mathbf{R}_{+} ; \mathbf{R}_{+}\right), d_{1}, d_{2}>0, \lambda_{1}, \lambda_{2} \in[0,1], \beta_{1}, \beta_{2} \geq 0$, and $u_{0}, v_{0} \in$ $\boldsymbol{L}_{+}^{\infty}(\Omega)$, have been studied by several authors; e.g. $[2,3,4,6,10]$. (Here we use $u, v$ rather than $U_{1}, U_{2}$ for notational convenience.) It follows from the results in Hollis, Martin, and Pierre [4] that $T_{\max }=\infty$ (and, moreover, that $u, v$ are uniformly bounded in $t$ ) under the following assumptions:

$$
\begin{gathered}
f(\xi) \leq c_{1}+c_{2} \xi^{\gamma} \text { for all } \xi \geq 0, \text { where } \gamma \geq 1 ; \\
0<\lambda_{1}, \lambda_{2}<1, \quad \lambda_{1}=\lambda_{2}=1, \quad \text { or } \quad \lambda_{1}=\lambda_{2}=\beta_{1}=\beta_{2}=0 .
\end{gathered}
$$

When $\beta_{1}=\beta_{2}=0$, assumption (1.4) may be dispensed with; that is, we may take $0 \leq \lambda_{1}, \lambda_{2} \leq 1$ (cf. Masuda [10]). One can also modify the argument in [4] slightly to handle $0 \leq \lambda_{1}, \lambda_{2}<1$ (Neumann/Robin conditions) and the case where $0 \leq \lambda_{1} \leq 1$ and $\lambda_{2}=1$ with $\beta_{2}>0$, provided that $\beta_{1}=0$ when $\lambda_{1}=0$. However, the situation where $\lambda_{1}=1, \beta_{1}>0$, and $\lambda_{2}=\beta_{2}=0$ (i.e., a Neumann condition for $v$ and a nonhomogeneous Dirichlet condition for $u$ ) presents fundamental difficulties. (See also the brief discussion of "critical" boundary conditions in Martin and Pierre [9].) It is precisely this type of situation that is the main focus of this note.

In [3] it is shown that the solution of (1.2) with $d_{1}>0$ and $d_{2}=0$ can blow up in finite time if $\lambda_{1}=1, \beta_{1}>0$, and $f(v)=v^{\gamma}$ with $\gamma>1$. To see this, suppose that $v_{0}$ is bounded away from zero near $x_{0} \in \partial \Omega$, and note that since $u=\beta_{1}>0$ on $\partial \Omega$, one has a lower bound $u \geq \frac{1}{2} \beta_{1}>0$ on some set $\mathscr{H}(t)=\left\{x \in \Omega:\left|x-x_{0}\right| \leq \varepsilon(t)\right\}$ for $t>0$ with $\varepsilon(t)>0$ so long as the solution exists. Now we see that $v_{t} \geq \frac{1}{2} \beta_{1} v^{r}$ on $\mathscr{H}(t)$, and thus assuming that the solution exists for all time leads to the obvious contradiction, since solutions of the equation $y^{\prime}(t)=\frac{1}{2} \beta_{1} y(t)^{\gamma}$ with $y(0)>0$ tend to infinity in finite time.

For the problem in the preceding paragraph, except with $d_{2}>0$ and $\lambda_{2}=\beta_{2}=0$, it has recently been shown by Bebernes and Lacey [1] that solutions can blow up in finite time if $\gamma>2$ and $\Omega$ is a ball in $\mathbf{R}^{n}, n \geq 1$. Note that this blowup may be directly attributed to the mixture of boundary condition types, since global existence is known for this problem with boundary conditions of uniform type. For $1<\gamma \leq 2$ 
and mixed boundary condition types, the question of global existence versus finitetime blowup remains open.

It is interesting to note also that if a solution of (1.2) does exist globally, mixed boundary condition types as those described above will cause the solution to grow without bound as $t \rightarrow \infty$. More precisely, we have the following result, the proof of which is due to Kirane [7].

Proposition 1.2. Consider problem (1.2) where $d_{1}, d_{2}>0, \lambda_{1}=1, \beta_{1}>0$, and $\lambda_{2}=\beta_{2}=0$ and where $f$ is nondecreasing with $f(\xi)>0$ for all $\xi>0$. If $(u, v)$ is a nonnegative solution on $\Omega \times\left[0, T_{\max }\right)$ with $v$ not identically zero, then $\int_{\Omega} v \geq a t+b$ for all $t \in\left[0, T_{\max }\right)$ where $a>0$ and $b \geq 0$.

Proof. Take $\zeta$ to be the solution of the elliptic problem

$$
\begin{aligned}
& -\Delta \zeta=1 \text { in } \Omega \text {, } \\
& \zeta=0 \text { on } \partial \Omega \text {, }
\end{aligned}
$$

and define $p(t)=\int_{\Omega}(\varepsilon \zeta u+v)$ where $\varepsilon>0$. Then we have

$$
\begin{aligned}
p^{\prime}(t) & =\int_{\Omega}\left(\varepsilon \zeta\left(d_{1} \Delta u-u f(v)\right)+d_{2} \Delta v+u f(v)\right) \\
& =\varepsilon d_{1} \int_{\Omega} \zeta \Delta u+\int_{\Omega}(1-\varepsilon \zeta) u f(v) \\
& =-\varepsilon d_{1} \beta_{1} \int_{\partial \Omega} \frac{\partial \zeta}{\partial n}-\varepsilon d_{1} \int_{\Omega} u+\int_{\Omega}(1-\varepsilon \zeta) u f(v) \\
& =\varepsilon d_{1} \beta_{1}|\Omega|+\int_{\Omega}\left((1-\varepsilon \zeta) f(v)-\varepsilon d_{1}\right) u .
\end{aligned}
$$

Note that if $f\left(v\left(\cdot, t_{0}\right)\right) \geq \varepsilon d_{1} /\left(1-\varepsilon\|\zeta\|_{\infty}\right)>0$, then $f(v(\cdot, t)) \geq \varepsilon d_{1} /\left(1-\varepsilon\|\zeta\|_{\infty}\right)$ for all $t \geq t_{0}$ since $f$ is nondecreasing and $v(x, t) \geq \min v\left(\cdot, t_{0}\right)>0$ for all $x \in \Omega$ and $t \geq t_{0}>0$. Thus by choosing $\varepsilon$ small enough, we can deduce that $p^{\prime}(t) \geq \varepsilon d_{1} \beta_{1}|\Omega|$ for all $t \geq t_{0}$ for some $t_{0} \geq 0$. It follows that $p(t) \geq \varepsilon d_{1} \beta_{1}|\Omega| t+c$, which yields the desired result.

REMARK. Note that this argument uses only that $f$ is a nondecreasing function; hence the result is true even for bounded nondecreasing functions $f$.

In [11] Morgan recently generalized the results of Hollis, Martin, and Pierre [4] to establish global existence for solutions of the $m$-component system (1.1), $m \geq 2$, where again $F$ is polynomially bounded (cf. (1.3)) and also satisfies an "Intermediate Sums" condition of the form

$$
A F(U) \leq C U+b \text { for all } U \in \mathbf{R}_{+}^{m}
$$

where $A$ and $C$ are $m \times m$ matrices with nonnegative entries, $A$ is lower triangular and nonsingular, and $b \in \mathbf{R}^{m}$. (The linear bound (1.5) may be relaxed somewhat to handle a polynomial bound of some small degree depending on the spatial dimension $n$; see [11].) Moreover, the assumption is also made that

$$
\begin{aligned}
\lambda_{i}=0 & \text { for all } i=1, \ldots, m, \\
0<\lambda_{i}<1 & \text { for all } i=1, \ldots, m, \\
\text { or } \quad \lambda_{i}=1 & \text { for all } i=1, \ldots, m .
\end{aligned}
$$


Minor modification of the arguments in [11] will allow the case in which $0 \leq \lambda_{i}<1$ for all $i=1, \ldots, m$ (a mixture of Neumann and Robin conditions), but again the case involving Neumann/Robin conditions mixed with nonhomogeneous Dirichlet conditions must, in general, be excluded.

In the section that follows, we will present some modest positive results concerning situations in which one can deduce global existence of solutions to (1.1) in spite of having a mixture of Neumann and nonhomogeneous Dirichlet boundary conditions.

2. Some positive results. We begin by partitioning the system (1.1) into a pair of coupled systems, with $l$ and $m-l$ components, respectively, of the form

$$
\begin{array}{ll}
\mathbf{u}_{t}-D_{1} \Delta \mathbf{u}=f(\mathbf{u}, \mathbf{v}) & \text { in } \Omega \times\{t>0\}, \\
B_{1} \mathbf{u}=\sigma & \text { on } \partial \Omega \times\{t>0\}, \\
\mathbf{u}(\cdot, 0)=\mathbf{u}_{0}(\cdot) & \text { on } \Omega ; \\
\mathbf{v}_{t}-D_{2} \Delta \mathbf{v}=g(\mathbf{u}, \mathbf{v}) & \text { in } \Omega \times\{t>0\}, \\
\mathbf{v}=\rho & \text { on } \partial \Omega \times\{t>0\}, \\
\mathbf{v}(\cdot, 0)=\mathbf{v}_{0}^{-}(\cdot) & \text { on } \Omega
\end{array}
$$

where $\mathbf{u}=\left(u_{j}\right)_{j=1}^{l}, \mathbf{v}=\left(v_{j}\right)_{j=1}^{m-l}, f=\left(f_{j}\right)_{j=1}^{l}, g=\left(g_{j}\right)_{j=1}^{m-l}, D_{1}=\operatorname{diag}\left\{d_{1}, d_{2}, \ldots\right.$, $\left.d_{l}\right\}$ with each $d_{j}>0$, and $D_{2}=\operatorname{diag}\left\{\delta_{1}, \delta_{2}, \ldots, \delta_{m-l}\right\}$ with each $\delta_{j}>0$. Also, $\sigma=\left(\sigma_{j}\right)_{j=1}^{l}$ and $\rho=\left(\rho_{j}\right)_{j=1}^{m-l}$, and the partitioning is done to accomplish separating boundary condition types according to

$$
B_{1} \mathbf{u}=\left(\eta_{j} u_{j}+\left(1-\eta_{j}\right) \frac{\partial u_{j}}{\partial n}\right)_{j=1}^{l} \quad \text { with each } \eta_{j} \in[0,1) .
$$

Also, $\mathbf{u}_{0}=\left(u_{0 j}\right)_{j=1}^{l}, \mathbf{v}_{0}=\left(v_{0 j}\right)_{j=1}^{m-l}$, and the basic properties $(\mathbf{H})$ are inherited throughout.

Proposition 2.1. Suppose that the functions $f$ and $g$ in $(2.1 . a, b)$ are polynomially bounded and satisfy

$$
\begin{aligned}
& \mathscr{A} f(\mathbf{u}, \mathbf{v}) \leq \mathscr{P} \mathbf{u}+\mathscr{Q} \mathbf{v}+a, \\
& \mathscr{B} g(\mathbf{u}, \mathbf{v}) \leq \mathscr{R} \mathbf{u}+\mathscr{S} \mathbf{v}+b
\end{aligned}
$$

for all $\mathbf{u} \in \mathbf{R}_{+}^{l}$ and $\mathbf{v} \in \mathbf{R}_{+}^{m-l}$, where $a$ and $b$ are vectors in $\mathbf{R}^{l}$ and $\mathbf{R}^{m-l}$, respectively, and

$\mathscr{A}, \mathscr{B}, \mathscr{P}$, and $\mathscr{S}$ are square matrices, $\mathscr{Q}$ and $\mathscr{R}$ are matrices with appropriate dimensions, and all have nonnegative entries.

Moreover, $\mathscr{A}$ and $\mathscr{B}$ are lower triangular and nonsingular.

Then, solutions of (2.1.a,b) (i.e., (1.1)) exist for all $t>0$; that is, $T_{\max }=\infty$.

Proposition 2.2. Suppose that the functions $f$ and $g$ in (2.1.a,b) are polynomially bounded and satisfy either

$$
\begin{aligned}
\mathscr{A} f(\mathbf{u}, \mathbf{v}) & \leq \mathscr{P} \mathbf{u}+a, \\
g(\mathbf{u}, \mathbf{v}) & \leq \mathscr{Q}(\mathbf{u}) \mathbf{v}+\mathscr{b}(\mathbf{u})
\end{aligned}
$$


for all $\mathbf{u} \in \mathbf{R}_{+}^{l}$ and $\mathbf{v} \in \mathbf{R}_{+}^{m-l}$ where

$\mathscr{A}$ and $\mathscr{P}$ are square matrices with nonnegative entries, $\mathscr{A}$ is lower triangular and nonsingular, $a \in \mathbf{R}^{l}$, and $\mathscr{Q}: \mathbf{R}^{l} \rightarrow \mathbf{R}^{(m-l) \times(m-l)}$ and $b: \mathbf{R}^{l} \rightarrow \mathbf{R}^{m-l}$ are polynomially bounded functions

or

$$
\begin{aligned}
f(\mathbf{u}, \mathbf{v}) & \leq \mathscr{Q}(\mathbf{v}) \mathbf{u}+b(\mathbf{v}), \\
\mathscr{A} g(\mathbf{u}, \mathbf{v}) & \leq \mathscr{P} \mathbf{v}+a
\end{aligned}
$$

for all $\mathbf{u} \in \mathbf{R}^{l}$ and $\mathbf{v} \in \mathbf{R}^{m-l}$ where $\mathscr{A}, \mathscr{P}, \mathscr{Q}, a$, and $b$ satisfy (2.5) with $l$ exchanged with $m-l$. Then solutions of $(2.1$.a,b) (i.e. (1.1)) exist for all $t>0$; that is, $T_{\max }=\infty$.

3. Proofs of Propositions 2.1 and 2.2. We assume that the reader is familiar with the standard notation and the theory of the Lebesgue spaces $\boldsymbol{L}^{p}(\Omega)$ and $\boldsymbol{L}^{p}(\Omega \times(0, T))$ as well as the basic ideas of Sobolev theory. Norms of vector-valued functions are defined by $\|\mathbf{z}\|=\left\|\sum_{i}\left|z_{i}\right|\right\|$ throughout the following.

The method of proof will be a variation on that of Morgan [11] and of Hollis and Morgan [5]. It is an extended version of a duality argument that was first applied to (two-component) reaction-diffusion systems by Hollis, Martin, and Pierre [4] and makes use of $\boldsymbol{L}^{p}$ regularity theory $(p>1)$ for linear parabolic equations [8; Chapter IV]. The proof of Lemma 3.2, in particular, is a straightforward adaptation of arguments in [11] and is essentially the same as that of [5, Lemma 3.3], where the method is applied to situations in which one or more diffusion coefficients are zero. For this reason the details of its proof will not be included here.

The heart of the proof of Proposition 2.1 is the following lemma.

Lemma 3.1. Let the conditions of Proposition 2.1 be fulfilled, and let $0<T<T_{\max }$ or $T=T_{\max }$ if $T_{\max }<\infty$. Then there exists a constant $C_{p}(T)$ such that for all $i=1, \ldots, l$ and $k=1, \ldots, m-l$ :

$$
\text { if } 1<p<\frac{n+2}{n} \text {, }
$$

then

$$
\begin{aligned}
& \left\|u_{i}\right\|_{p, \Omega \times(0, T)} \leq C_{p}(T)\left[1+\|\mathbf{u}(\cdot, 0)\|_{p, \Omega}+\|\mathbf{u}\|_{1, \Omega \times(0, T)}+\|\mathbf{v}\|_{1, \Omega \times(0, T)}\right], \\
& \left\|v_{k}\right\|_{p, \Omega \times(0, T)} \leq C_{p}(T)\left[1+\|\mathbf{v}(\cdot, 0)\|_{p, \Omega}+\|\mathbf{u}\|_{1, \Omega \times(0, T)}+\|\mathbf{v}\|_{1, \Omega \times(0, T)}\right] ;
\end{aligned}
$$
and

$$
\text { if } \frac{n+2}{n+1}<p<\infty \text { and } r=\frac{p(n+2)}{p+n+2},
$$

then

$$
\begin{gathered}
\left\|u_{i}\right\|_{p, \Omega \times(0, T)} \leq C_{p}(T)\left[1+\|\mathbf{u}(\cdot, 0)\|_{p, \Omega}+\|\mathbf{u}\|_{r, \Omega \times(0, T)}+\|\mathbf{v}\|_{r, \Omega \times(0, T)}\right], \\
\left\|v_{k}\right\|_{p, \Omega \times(0, T)} \leq C_{p}(T)\left[1+\|\mathbf{u}(\cdot, 0)\|_{p, \Omega}+\|\mathbf{u}\|_{r, \Omega \times(0, T)}+\|\mathbf{v}\|_{r, \Omega \times(0, T)}\right] .
\end{gathered}
$$

Proof. The proof is a straightforward modification of the arguments in Morgan [11] and, in particular, Hollis and Morgan [5, Lemma 3.3]. 
Lemma 3.1 provides a bootstrapping mechanism for obtaining $\boldsymbol{L}^{p}$ estimates on $\mathbf{u}$ and $\mathbf{v}$ for arbitrary $p \in[1, \infty)$. To initiate this bootstrapping procedure, we need $L^{1}$ estimates, which are provided by

Lemma 3.2. Let the conditions of Proposition 2.1 be fulfilled, and let $0<T<T_{\max }$ or $T=T_{\max }$ if $T_{\max }<\infty$. Then there exists a constant $C(T)$ such that

$$
\|\mathbf{u}\|_{1, \Omega \times(0, T)}+\|\mathbf{v}\|_{1, \Omega \times(0, T)} \leq C(T) .
$$

Proof. First note that because of the assumptions on the matrices $\mathscr{A}$ and $\mathscr{B}$ in (2.2)-(2.3), we may assume without loss that $a_{l, j}>0$ for $j=1, \ldots, l$ and $b_{m-l, j}>0$ for $j=1, \ldots, m-l$. Let us now define

$$
w=\sum_{j=1}^{l} a_{l, j} u_{j} \quad \text { and } \quad z=\sum_{j=1}^{m-l} \delta_{j} b_{m-l, j} v_{j} .
$$

By (2.2) we have, for an appropriate constant $C_{1}$,

$$
\begin{gathered}
\sum_{j=1}^{l} a_{l, j} f_{j}(\mathbf{u}, \mathbf{v}) \leq C_{1}[1+w+z], \\
\sum_{j=1}^{m-l} b_{m-l, j} g_{j}(\mathbf{u}, \mathbf{v}) \leq C_{1}[1+w+z] .
\end{gathered}
$$

Applying (3.6), we see that $w_{t} \leq \sum_{j=1}^{l} d_{j} a_{l, j} \Delta u_{j}+C_{1}[1+w+z]$, and hence

$$
\int_{\Omega} w \leq \int_{\Omega} w(\cdot, 0)+\int_{0}^{t} \sum_{j=1}^{l} d_{j} a_{l, j} \int_{\partial \Omega} \frac{\partial u_{j}}{\partial n}+C_{1}\left[t+\int_{0}^{t} \int_{\Omega} w+\int_{0}^{t} \int_{\Omega} z\right] .
$$

Since $\partial u_{j} / \partial n=\left(\left(\sigma_{j}-\eta_{j} u_{j}\right) /\left(1-\eta_{j}\right)\right) \leq \sigma_{j} /\left(1-\eta_{j}\right)$ on $\partial \Omega$, we have

$$
\int_{\Omega} w \leq \int_{\Omega} w(\cdot, 0)+C_{2}\left[t+\int_{0}^{t} \int_{\Omega} w+\int_{0}^{t} \int_{\Omega} z\right] .
$$

Now define

$$
\zeta(\cdot, t)=\int_{0}^{t} z(\cdot, s) d s=\int_{0}^{t} \sum_{j=1}^{m-l} \delta_{j} b_{m-l, j} v_{j}(\cdot, s) d s .
$$

A straightforward calculation using (3.7) shows that

$$
\zeta_{t} \leq \delta_{\max } \Delta \zeta+C_{3}\left(z(\cdot, 0)+t+\int_{0}^{t} w+\zeta\right)
$$

A maximum principle argument now produces the estimate

$$
\zeta(\cdot, t)=\int_{0}^{t} z(\cdot, s) d s \leq C_{4} e^{\lambda t}\left(1+t+\int_{0}^{t} w\right)
$$

if $\lambda>C_{3}$. Returning to (3.8), we now see that

$$
\int_{\Omega} w \leq \int_{\Omega} w(\cdot, 0)+C_{5}(t)\left(1+t+\int_{0}^{t} \int_{\Omega} w\right),
$$


from which Gronwall's inequality yields a bound $\int_{\Omega} w(\cdot, t) \leq M_{1}(t)$ where $M_{1} \in$ $C\left(\mathbf{R}_{+} ; \mathbf{R}_{+}\right)$. Finally, (3.9) now implies a bound $\int_{0}^{t} \int_{\Omega} z \leq M_{2}(t)$ with $M_{2} \in$ $C\left(\mathbf{R}_{+} ; \mathbf{R}_{+}\right)$. From these last two inequalities follows the conclusion of the lemma.

We are now in a position to give the

Proof of Proposition 2.1. Let us assume for the sake of contradiction that $T_{\max }<$ $\infty$ in Proposition 1.1. The result of Lemma 3.2 together with (3.2) from Lemma 3.1 implies that if $p \in[1,(n+2) / n)$ then $u_{i}, v_{k} \in L^{p}\left(\Omega \times\left(0, T_{\max }\right)\right)$ for each $i=1, \ldots, l$ and $k=1, \ldots, m-l$. Taking $r=(n+2) /(n+a)$ in (3.3) results in $p=(n+2) r /(n+2-r)=(n+2) /(n+a-1)$, and with $\left\{a_{j}\right\}_{j=1}^{\infty}$ any decreasing sequence in $(1-n, 1]$ such that $a_{0}=1, a_{j}-a_{j+1} \leq 1$, and $a_{j} \rightarrow 1-n$ as $j \rightarrow \infty$, we have $r_{j}<r_{j+1} \leq p_{j}<p_{j+1} \rightarrow \infty$. From (3.2) and (3.3) we then obtain by induction that $u_{i}, v_{k} \in L^{p_{j}}\left(\Omega \times\left(0, T_{\max }\right)\right), j=1,2, \ldots$, for each $i$ and $k$, from which it follows by Sobolev imbedding (due to the polynomial growth assumptions on $f$ and $g)$ that $u_{i}, v_{k} \in L^{\infty}\left(\Omega \times\left(0, T_{\max }\right)\right)$ for each $i$ and $k$. Thus Proposition 1.1 leads by contradiction to the conclusion that $T_{\max }=\infty$.

The proof of Proposition 2.2 is similar to the above proof of Proposition 2.1. We will sketch the argument in the following.

Indication of the Proof of Proposition 2.2. Let us assume that the conditions (2.4) and (2.5) are met. Arguments similar to those that prove Lemmas 3.1 and 3.2 produce the following estimates for $T=T_{\max }$ if $T_{\max }<\infty$ :

$$
\begin{gathered}
\text { If } 1<p<\frac{n+2}{n}, \text { then for each } i=1, \ldots, l, \\
\left\|u_{i}\right\|_{p, \Omega \times(0, T)} \leq C_{p}(T)\left[1+\|\mathbf{u}(\cdot, 0)\|_{p, \Omega}+\|\mathbf{u}\|_{1, \Omega \times(0, T)}\right] . \\
\text { If } \frac{n+2}{n+1}<p<\infty \text { and } r=\frac{p(n+2)}{p+n+2}, \text { then for each } i=1, \ldots, l, \\
\left\|u_{i}\right\|_{p, \Omega \times(0, T)} \leq C_{p}(T)\left[1+\|\mathbf{u}(\cdot, 0)\|_{p, \Omega}+\|\mathbf{u}\|_{r, \Omega \times(0, T)}\right] . \\
\|\mathbf{u}\|_{1, \Omega \times(0, T)} \leq C(T) .
\end{gathered}
$$

From these, the bootstrapping procedure in the proof of Proposition 2.1 provides the estimates

$$
\|\mathbf{u}\|_{p, \Omega \times(0, T)} \leq C_{p}(T), \quad 1 \leq p<\infty .
$$

It now follows from assumption (2.4.b) and the results of Chapter VII of Ladyzhenskaya et al. [8], that $\mathbf{v} \in \boldsymbol{L}^{\infty}\left(\Omega \times(0, T) ; \mathbf{R}^{m-l}\right)$. Now, Sobolev imbedding puts $\mathbf{u} \in \boldsymbol{L}^{\infty}\left(\Omega \times(0, T) ; \mathbf{R}^{l}\right)$ as well. It therefore follows that $T_{\max }=\infty$. The proof is similar under the condition (2.6).

4. Applications. In this section we will examine some particular chemical reaction models in order to illustrate the applicability of Proposition 2.1. Throughout this section we will assume that all reactions take place in a bounded domain $\Omega$ with smooth boundary $\partial \Omega$. When we say that two functions $\xi_{1}$ and $\xi_{2}$ satisfy the same type of boundary condition, we mean that either both satisfy nonnegative Dirichlet conditions $\xi_{i}=\sigma_{i}$ on $\partial \Omega \times\{t>0\}$ or else both satisfy Robin/Neumann conditions $\partial \xi_{i} / \partial n=k_{i}\left(\sigma_{i}-\xi_{i}\right)$ on $\partial \Omega \times\{t>0\}$ where $\sigma_{i}, k_{i} \geq 0$. 
Let us begin with the simple three-component reaction $A+B \leftrightarrows C$ that leads (with unit reaction rates) to the reaction-diffusion system

$$
\begin{aligned}
& a_{t}-d_{1} \Delta a=-a b+c, \\
& b_{t}-d_{2} \Delta b=-a b+c, \\
& c_{t}-d_{3} \Delta c=a b-c .
\end{aligned}
$$

If boundary conditions of the same type are imposed on all three components of this system, then it follows from known results [11] that, for any nonnegative $L^{\infty}$ initial data, solutions of (4.1) exist for all $t>0$. It follows from Proposition 2.1 that solutions of (4.1) with nonnegative $\boldsymbol{L}^{\infty}$ initial data exist for all $t>0$ if $c$ satisfies the same type of boundary condition as either $a$ or $b$. Thus if boundary conditions of different types are imposed on $a$ and $b$, global existence follows regardless of the type of boundary condition that is imposed on $c$. To see this, consider, for example, the case where $a$ satisfies a Dirichlet boundary condition and $b$ and $c$ satisfy Neumann/Robin conditions. Then we take $\mathbf{u}=(b, c)^{\mathrm{T}}$ and $\mathbf{v}=(a)$ and observe that (2.2.a) and (2.2.b) are satisfied if we set $\mathscr{A}=\left[\begin{array}{ll}1 & 0 \\ 1 & 1\end{array}\right], \mathscr{P}=\left[\begin{array}{ll}0 & 1 \\ 0 & 0\end{array}\right]$, $\mathscr{Q}=[0], a=\left[\begin{array}{l}0 \\ 0\end{array}\right], \mathscr{B}=[1], \mathscr{R}=[0,1], \mathscr{S}=[0]$, and $b=[0]$.

We next consider a model of the oxygenation of hemoglobin ( $\mathrm{Hb})$ in a pulmonary capillary (see $[14,12])$. If we let $A, B, C, D$, and $E$ represent $\mathrm{Hb}, \mathrm{O}_{2}, \mathrm{HbO}_{2}$, $\mathrm{CO}_{2}$, and $\mathrm{HbCO}_{2}$, respectively, then the reactions considered are

$$
A+B \leftrightarrows C, \quad A+D \leftrightarrows E
$$

This leads to the five-component reaction-diffusion system:

$$
\begin{aligned}
& A_{t}-d_{1} \Delta A=-k_{1} A B+k_{2} C-k_{3} A D+k_{4} E, \\
& B_{t}-d_{2} \Delta B=-k_{1} A B+k_{2} C, \\
& C_{t}-d_{3} \Delta C=k_{1} A B-k_{2} C, \\
& D_{t}-d_{4} \Delta D=-k_{3} A D+k_{4} E, \\
& E_{t}-d_{5} \Delta E=k_{3} A D-k_{4} E .
\end{aligned}
$$

The result of Proposition 2.1 applied to this system is summarized as

Proposition 4.1. Solutions of (4.2) with nonnegative $L^{\infty}$ initial data exist for all $t>0$ provided that: (1) $C$ satisfies the same type of boundary condition as either $A$ or $B$, and (2) $E$ satisfies the same type of boundary condition as either $A$ or $D$.

Note that this covers the physically interesting case in which $\partial A / \partial n=\partial C / \partial n=$ $\partial E / \partial n=0, B=\beta$, and $D=\gamma$ on $\partial \Omega \times\{t>0\}$. For the sake of illustration, we will indicate for this case how the assumptions of Proposition 2.2 are verified. If we define $\mathbf{u}=(A, C, E)^{\mathrm{T}}$ and $\mathbf{v}=(B, D)^{\mathrm{T}}$, then (2.2.a) is satisfied with $a=0$ and the matrices $\mathscr{A}, \mathscr{P}$, and $\mathscr{Q}$ taken to be

$$
\left[\begin{array}{lll}
1 & 0 & 0 \\
1 & 1 & 0 \\
1 & 1 & 1
\end{array}\right], \quad\left[\begin{array}{ccc}
0 & k_{2} & k_{4} \\
0 & 0 & k_{4} \\
0 & 0 & 0
\end{array}\right], \quad \text { and }\left[\begin{array}{ll}
0 & 0 \\
0 & 0 \\
0 & 0
\end{array}\right] \text {, }
$$


respectively. Also, (2.2.b) is satisfied if we take $b=0$ and the matrices $\mathscr{B}, \mathscr{R}$, and $\mathscr{S}$ to be

$$
\left[\begin{array}{ll}
1 & 0 \\
0 & 1
\end{array}\right], \quad\left[\begin{array}{ccc}
0 & k_{2} & 0 \\
0 & 0 & k_{4}
\end{array}\right], \quad \text { and } \quad\left[\begin{array}{ll}
0 & 0 \\
0 & 0
\end{array}\right] \text {, }
$$

respectively.

Another model, which is very similar to the preceding one, is the following model of phosphorus diffusion in silicon [13, 5]:

$$
V+I \leftrightarrows\langle 0\rangle, \quad P+V \leftrightarrows E, \quad P+I \leftrightarrows F
$$

where $P$ represents a substitutional phosphorus atom, $I$ an interstitial site, $V$ a lattice vacancy, $E$ a phosphorus-vacancy pair, and $F$ a phosphorus-interstitial pair. This leads to the reaction-diffusion system:

$$
\begin{aligned}
V_{t}-d_{1} \Delta V & =-k_{1} P V+k_{2} E-k_{0}\left(V I-V_{e q} I_{e q}\right), \\
E_{t}-d_{2} \Delta E & =k_{1} P V-k_{2} E, \\
I_{t}-d_{3} \Delta I & =-k_{3} P I+k_{4} F-k_{0}\left(V I-V_{e q} I_{e q}\right), \\
F_{t}-d_{4} \Delta F & =k_{3} P I-k_{4} F, \\
P_{t}-d_{5} \Delta P & =-k_{1} P V+k_{2} E-k_{3} P I+k_{4} F
\end{aligned}
$$

where the $k_{i}$ are positive reaction rates and the $d_{i}$ are positive diffusion coefficients. In $[13,5]$ the diffusion coefficient $d_{5}$ is taken to be zero, reflecting the assumption that the only significant transport mechanism for phosphorus is the combination with the vacancies and interstitials; however, for our purposes here, we will assume $d_{5}>0$ in analogy with the idea of artificial viscosity. Of physical interest for this model (cf. [13]) are the boundary conditions:

$$
\begin{aligned}
& V=V_{e q}, \quad I=I_{e q}, \\
& \frac{\partial E}{\partial n}=\frac{\partial F}{\partial n}=0 \quad \text { on } \partial \Omega \times\{t>0\}, \\
& \frac{\partial P}{\partial n}=k\left(C^{*}-P\right) .
\end{aligned}
$$

By defining $\mathbf{u}=(P, E, F)^{\mathrm{T}}$ and $\mathbf{v}=(V, I)^{\mathrm{T}}$, we see that Proposition 2.1 guarantees the existence of global solutions for any nonnegative $\boldsymbol{L}^{\infty}$ initial data. Other combinations of boundary condition types are also covered by Proposition 2.1; they are summarized in the following proposition.

Proposition 4.2. Solutions of (4.3) with nonnegative $L^{\infty}$ initial data exist for all $t>0$ provided that: (1) $E$ satisfies the same type of boundary condition as either $V$ or $P$, and (2) $F$ satisfies the same type of boundary condition as either $I$ or $P$. We conclude with an illustration of Proposition 2.2. Let us consider the system

$$
\begin{aligned}
u_{t}-d_{1} \Delta u & =-u^{\alpha} v^{\beta}-u^{\gamma} w^{\rho}+\lambda_{1} v+\lambda_{2} w \\
v_{t}-d_{2} \Delta v & =-u^{\alpha} v^{\beta}+u^{\gamma} w^{\rho} \\
w_{t}-d_{3} \Delta w & =u^{\alpha} v^{\beta}+u^{\gamma} w^{\rho}
\end{aligned}
$$


where $\alpha, \beta, \gamma, \rho \geq 1$ and $\lambda_{1}, \lambda_{2} \geq 0$. Unless all three boundary conditions are of the same type, Proposition 2.1 does not apply here. However, the result of Proposition 2.2 applied to (4.4) may be summarized as

Proposition 4.3. Solutions of (4.4) with nonnegative $L^{\infty}$ initial data exist for all $t>0$ provided that either: (1) $\lambda_{1}=0$ and $u$ satisfies the same type of boundary condition as $w$, or else (2) $\lambda_{2}=0, \rho=1$, and $u$ satisfies the same type of boundary condition as $v$.

\section{REFERENCES}

[1] J. Bebernes and A. Lacey, Finite time blowup for semilinear reactive-diffusive systems, J. Differential Equations 95, 105-129 (1992)

[2] A. Haraux and A. Youkana, On a result of $K$. Masuda concerning reaction-diffusion equations, Tohoku Math. J. 40, 159-163 (1988)

[3] S. Hollis, Globally bounded solutions of reaction-diffusion systems, Ph.D. Thesis, North Carolina State Univ., 1986

[4] S. Hollis, R. Martin, and M. Pierre, Global existence and boundedness in reaction-diffusion systems, SIAM J. Math. Anal. 18, 744-761 (1987)

[5] S. Hollis and J. Morgan, Partly dissipative reaction-diffusion systems and a model of phosphorus diffusion in silicon, J. Nonlinear Analysis-Theory, Methods and Applications, in press.

[6] M. Kirane, Global bounds and asymptotics for a system of reaction-diffusion equations, J. Math. Anal. Appl. 138, 328-342 (1989)

[7] M. Kirane, private communication, 1987

[8] O. A. Ladyzhenskaya, V. A. Solonnikov, and N. N. Ural'ceva, Linear and quasilinear equations of parabolic type, Transl. Math. Monographs, vol. 23, Amer. Math. Soc., Providence, RI, 1968

[9] R. Martin and M. Pierre, Nonlinear reaction-diffusion systems, Nonlinear Equations in the Applied Sciences, edited by W. F. Ames and C. Rogers, Academic Press (to appear)

[10] K. Masuda, On the global existence and asymptotic behavior of solutions of reaction-diffusion systems, Hokkaido Math. J. 12, 360-370 (1982)

[11] J. Morgan, Global existence for semilinear parabolic systems, SIAM J. Math. Anal. 20, 1128-1144 (1989)

[12] J. Morgan, Boundedness and decay results for reaction-diffusion systems, SIAM J. Math. Anal. 21, $1172-1189$ (1990)

[13] W. B. Richardson and B. J. Mulvaney, Plateau and kink in P profiles diffused into Si: A result of strong bimolecular recombination?, Appl. Phys. Lett. 53, 1917-1919 (1988)

[14] M. Singh, K. Khetarpal, and M. Sharan, $A$ theoretical model for studying the rate of oxygenation of blood in pulmonary capillaries, J. Math. Biol. 9, 305-330 (1980) 